\title{
Supporting Information for: Carrier diffusion lengths exceeding 1 micron despite trap-limited transport in halide double perovskites
}

Milan Delor ${ }^{1, \#}$, Adam H. Slavney ${ }^{2}$, Nathan R. Wolf ${ }^{2}$, Marina R. Filip ${ }^{3,4}$, Jeffrey B. Neaton ${ }^{3,5,6}$, Hemamala I. Karunadasa2, Naomi S. Ginsberg ${ }^{1,3,5,6,7,8}$

${ }^{1}$ Department of Chemistry, University of California Berkeley, Berkeley, CA, USA

${ }^{2}$ Department of Chemistry, Stanford University, Stanford, CA, USA

${ }^{3}$ Department of Physics, University of California Berkeley, Berkeley, CA, USA

${ }^{4}$ Molecular Foundry, Lawrence Berkeley National Lab, Berkeley, CA, USA

${ }^{5}$ Materials Sciences Division, Lawrence Berkeley National Lab, Berkeley, CA, USA

${ }^{6}$ Kavli Energy NanoSciences Institute at Berkeley, Berkeley, CA, USA

${ }^{7}$ STROBE, National Science Foundation Science and Technology Center, University of California Berkeley, Berkeley, CA, USA

${ }^{8}$ Molecular Biophysics and Integrated Bioimaging Division, Lawrence Berkeley National Laboratory, Berkeley, CA, USA

\# Present address: Department of Chemistry, Columbia University, New York, NY, USA

\section{Materials and methods}

Safety warning: Salts of $\mathrm{T}^{3+}$ are toxic, with a multitude of acute and chronic deleterious health effects, and reactions involving these ions should be undertaken with proper precautions. Common signs of acute $\mathrm{Tl}$ exposure include constipation, swelling and loss of feeling in the extremities, and hair loss. If exposure is suspected, medical attention should be sought immediately. $\mathrm{Tl}^{3+}$ may be absorbed via inhalation, ingestion, or through skin contact. Proper personal protective equipment should be worn at all times including solvent-compatible gloves. Particular care should be taken when working with $\mathrm{TI}^{3+}$ and nonaqueous solvents particularly acetone, dimethylformamide (DMF), and dimethylsulfoxide (DMSO) all of which can both dissolve significant amounts of $\mathrm{TIX}_{3}$ and penetrate nitrile gloves. Manipulation of fine powders should be performed in a fume hood.

In our laboratory, we have established several safety guidelines to minimize the risk of Tl exposure. We have designated a specific workspace for reactions with these salts and have separate waste streams for them. Aluminum foil is laid out on the work surface prior to all manipulations to contain any spills and discarded afterwards as contaminated waste. Gloves are changed after manipulating TI-containing solids or solutions. When possible, we use disposable lab supplies for these reactions, which are discarded immediately after use. When this is not possible, we have designated equipment for use only with $\mathrm{TI}$ containing reactions. If glassware cannot be disposed, it is cleaned by treatment with aqua regia followed by successive rinses with water, dilute ammonium hydroxide (to remove trace $\mathrm{AgX}$ ), water, then isopropanol. Cleaning glassware with acetone should be avoided due to the risks of $\mathrm{Tl}^{3+}$ exposure. 
Synthesis: Single crystals of $\mathrm{Cs}_{2} \mathrm{AgBiBr}_{6}(\mathrm{Ag}-\mathrm{Bi})$ and $\mathrm{Cs}_{2} \mathrm{AgTlBr}_{6}$ (Ag-TI) were synthesized according to previously-published procedures: see supporting ref 1 for $\mathrm{Ag}-\mathrm{Bi}$, and supporting ref 2 for $\mathrm{Ag}$-Tl. Solvents were of reagent grade or higher purity. All reagents were purchased from commercial vendors and used as received.

For $\mathrm{Ag}-\mathrm{Bi}, \mathrm{CsBr}(0.426 \mathrm{~g}, 2.00 \mathrm{mmol})$ and $\mathrm{BiBr}_{3}(0.449 \mathrm{~g}, 1.00 \mathrm{mmol})$ were dissolved in $10 \mathrm{~mL}$ of $9 \mathrm{M}$ concentrated $\mathrm{HBr}$ in a $20 \mathrm{~mL}$ glass vial. $\mathrm{AgBr}(0.188 \mathrm{~g}, 1.00 \mathrm{mmol})$ was then added to the solution and the vial was capped and heated to $110{ }^{\circ} \mathrm{C}$. The solution was held at $110{ }^{\circ} \mathrm{C}$ for $2 \mathrm{~h}$ and cooled to room temperature at a rate of $1{ }^{\circ} \mathrm{C} / \mathrm{hr}$, yielding red truncated octahedral crystals. Single crystals were isolated from solution by filtration on glass fiber filter paper and stored in an $\mathrm{N}_{2}$-sparged vial.

For $\mathrm{Ag}-\mathrm{Tl}, \mathrm{CsBr}(0.533 \mathrm{~g}, 2.50 \mathrm{mmol}), \mathrm{AgBr}(0.939 \mathrm{~g}, 5.00 \mathrm{mmol})$, and $\mathrm{Tl}_{2} \mathrm{O}_{3}(0.285 \mathrm{~g}, 0.625 \mathrm{mmol})$ were combined in $20 \mathrm{~mL}$ of $9 \mathrm{M}$ concentrated $\mathrm{HBr}$ in a $20 \mathrm{~mL}$ glass vial and sealed with a polystyrene cap. The vial was heated to $100{ }^{\circ} \mathrm{C}$ for ca. $2 \mathrm{~h}$ until the solids had fully dissolved, giving a pale yellow solution. The vial was slowly cooled to room temperature at a rate of $1{ }^{\circ} \mathrm{C} / \mathrm{hr}$, yielding black truncated octahedral crystals. Single crystals were isolated from solution by filtration on glass fiber filter paper and stored in an $\mathrm{N}_{2}$-sparged vial.

Sample handling: For stroboSCAT measurements, the mm-scale single crystals are mounted on glass coverslips, and a thin layer of PMMA dissolved in toluene was drop-cast on top of the single crystals to protect them, hold them in place, and provide a refractive-index matched environment. For cleavedsurface measurements of Ag-Bi crystals, the crystals are cleaved using an $\mathrm{x}$-acto knife blade in a nitrogen glovebox $\left(<1 \mathrm{ppm} \mathrm{O}_{2}\right.$ and $\mathrm{H}_{2} \mathrm{O}$ ). The crystals are then mounted cleaved-surface down on glass coverslips, PMMA is immediately drop-cast on top, and stroboSCAT measurements are completed within 3 hours of cleaving. Thermal annealing on Ag-Tl crystals is performed on a hot plate in a nitrogen glovebox $(<1 \mathrm{ppm}$ $\mathrm{O}_{2}$ and $\mathrm{H}_{2} \mathrm{O}$ ) at $100{ }^{\circ} \mathrm{C}$ for 4 days. The crystals are already mounted on a glass substrate and are surrounded by PMMA. The hot plate is set to $105-110^{\circ} \mathrm{C}$ and the temperature on top of the substrate is periodically monitored to remain at $100 \pm 5^{\circ} \mathrm{C}$.

Trap density estimations are performed assuming that the turnover time $\tau_{\text {turnover }}$ occurs when the carrier density $\left(n_{\mathrm{c}}\right)$ is equal to the trap density ( $\left.n_{\text {traps }}\right)$. The task is then to estimate $n_{\mathrm{c}}$ at $t=\tau_{\text {turnover }}$. To do so, we need to account for carrier recombination and diffusion out of the initially-excited volume. For simplicity, we assume the initial volume is a hemi-sphere of radius $r$ whose magnitude is dictated by the pump spot size, and that carrier diffusion occurs isotropically in all directions within the crystal. Thus, the volume at time $\tau_{\text {turnover }}$ is $V_{0} \times\left[r\left(t=\tau_{\text {turnover }}\right) / r\left(t=t_{0}\right)\right]^{3}$, where $V_{0}$ is the volume at time $t=t_{0}$, i.e. immediately following pump excitation. We extract $r\left(t=\tau_{\text {turnover }}\right)$ and $r\left(t=t_{0}\right)$ from the raw stroboSCAT data. The carrier density (and therefore trap density) at time $\tau_{\text {turnover }}$ is then

$$
n_{c, t=\tau(\text { turnover })}=n_{\text {traps }}=n_{c, t=t(0)} \times f \times\left[\frac{r\left(t=t_{0}\right)}{r\left(t=\tau_{\text {turnover }}\right)}\right]^{3},
$$

where $f$ is the empirically-measured fraction of carrier population remaining after time $\tau_{\text {turnover, }}$ thus accounting for carrier recombination (i.e. $f=0.7$ if $30 \%$ of carriers have recombined), and $n_{c, t=t(0)}$ is the known, pump-fluence-dependent carrier density at time $t=0$.

These simple estimations are subject to a caveat: as shown in Figure 3 of the main text, bulk mobilities may be generally different than near-surface mobilities. As such, $r\left(t=\tau_{\text {turnover }}\right)$, which we empirically measure only near the surface of the crystal, may not represent the true average distribution of carriers 
throughout the crystal, which will result in our case to tend toward overestimating the trap densities. For $\mathrm{Ag}-\mathrm{Bi}$, where we have knowledge of both near-surface and bulk mobilities in the saturated-trap regime, we estimate a lower and higher bound for the trap densities: For the two limiting cases of bulk only and near-surface only, the calculated trap densities differ by a factor of 4 when comparing $r$ at the turnover time of $15 \mathrm{~ns}$ using $D_{\text {saturated }}=0.16$ or $0.4 \mathrm{~cm}^{2} / \mathrm{s}$ for near-surface and bulk, respectively. Given the pump penetration depth of $35 \mathrm{~nm}$, and assuming the crystal is fully bulk-like between 100-200 nm below the surface, we estimate that, on average, about half the carrier density will be evolving in the bulk environment rather than the near-surface environment during the first $15 \mathrm{~ns}$. Therefore, compounding the factor of 4 with a split density between near-surface and bulk carriers, we can estimate a lower bound for the trap density to be half of that calculated using only near-surface behavior. We therefore report the range for the trap density near the Ag-Bi surface to be $1-2 \times 10^{17} \mathrm{~cm}^{-3}$. Diffusion lengths under solar fluences are estimated using $L_{d}=\sqrt{2 D_{\text {trapped }} \tau}$, where $\tau$ is the measured major component of the carrier lifetime. Indeed, at solar fluences $\left(n_{0} \approx 10^{13}-10^{14} \mathrm{~cm}^{-3}\right)$ in the measured single crystals of $\mathrm{Ag}-\mathrm{Bi}$ and $\mathrm{Ag}-\mathrm{Tl}$, traps are not saturated, so that all charges can be shallow-trapped, similarly to the late-time or lowdensity regimes in our data. We therefore use the slower $D_{\text {trapped }}$ extracted from our measurements at late time delays, as opposed to a combination of $D_{\text {saturated }}$ and $D_{\text {trapped, }}$, to estimate carrier diffusion lengths under solar fluences. We make the assumption, supported by bulk transport measurements in the literature and by our lowest-excitation/carrier density data, that deep trap densities are extremely low in these materials (typically reported as $<10^{10} \mathrm{~cm}^{-3}$ ) 3 and therefore will not affect carrier dynamics at solar fluences. Under this assumption, diffusion lengths should not change for carrier densities ranging from $10^{10} \mathrm{~cm}^{-3}$ to $\sim 10^{16} \mathrm{~cm}^{-3}$, and the correct diffusivity to use is $D_{\text {trapped }}$ as measured in our data. Above a carrier density of $\sim 10^{16} \mathrm{~cm}^{-3}$, trap saturation would lead to larger diffusion lengths.

stroboSCAT measurements were performed on an instrument described elsewhere in extensive detail ${ }^{4}$. The setup is illustrated in Figure S1. Briefly, pulsed laser diodes (LDH-series, PicoQuant, pulsewidth 100 ps FWHM) at 440, 470 and $520 \mathrm{~nm}$ (pump) and $640 \mathrm{~nm}$ (probe) were used as light sources. For the experiments shown in the text, we use a base laser repetition rate of $250 \mathrm{kHz}$ or $500 \mathrm{kHz}$. The pump is modulated at $660 \mathrm{~Hz}$. Pump-probe delay times are controlled using the electronic delay capabilities of the laser driver with $20 \mathrm{ps}$ resolution.

Both pump and probe are spatially-filtered through $20 \mu \mathrm{m}$ pinholes (SF) and telescoped to 6 $\mathrm{mm}$ and 1 $\mathrm{mm}$ beam diameters, respectively, before entering the microscope. The two beams are combined using a longpass filter and are directed to a home-built microscope of very similar design to the microscope body detailed in Ortega Arroyo et al. $.^{5} \mathrm{~A} f=300 \mathrm{~mm}$ wide-field lens (WFL) is inserted in the probe beam path upstream of the LP to focus the beam in the back focal plane of the objective, resulting in wide-field illumination ( $20 \mu \mathrm{m}$ depending on the beam size prior to the WFL) of the sample. The pump is sent collimated into the objective, resulting in a focused excitation spot of $306 \mathrm{~nm}$ FWHM. A 50/50 beamsplitter (BS) reflects the pump and probe light into a high numerical aperture (1.4 NA) oil-immersion objective (Leica HC PL APO 63x/1.40NA) and onto the sample. Probe light reflected from the samplesubstrate interface as well as scattered from the sample are collected through the same objective. The light transmitted through the beamsplitter is focused onto a complementary metal-oxide semiconductor (CMOS) detector (PixeLINK PL-D752, equipped with the Sony IMX 174 global shutter sensor) using a f=500 $\mathrm{mm}$ lens placed one tube length $(200 \mathrm{~mm})$ away from the back focal plane of the objective. The total magnification is $157.5 \mathrm{x}$, and the instrument response function is $\sim 240$ ps FWHM. 


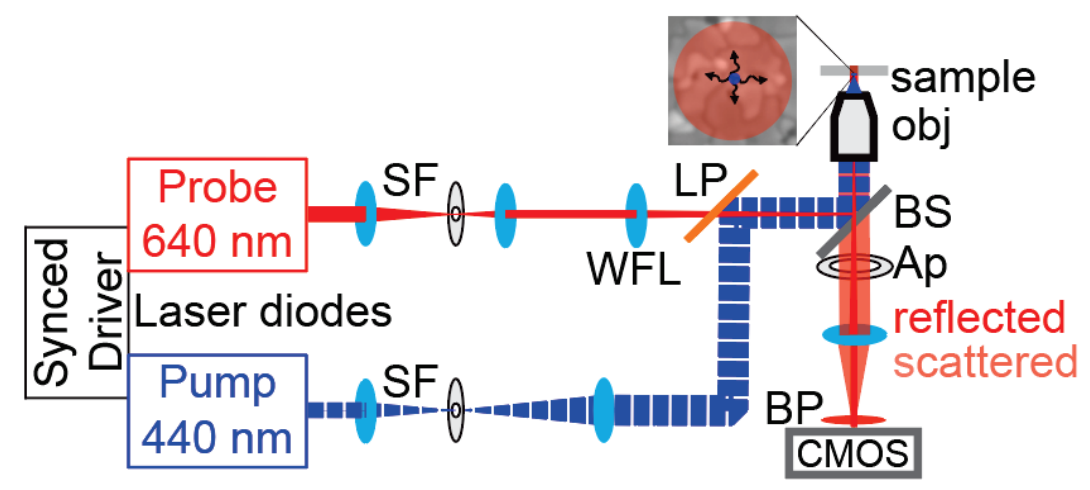

Figure S1. Schematic of the stroboSCAT setup. Laser diodes (PicoQuant) synchronized through a single oscillator/laser driver are used for pump and probe, using driver electronics to delay the probe with respect to the pump. After passing through spatial filters (SF), the beams are combined with a longpass filter (LP) before reflecting off a $50 / 50$ beamsplitter (BS) into the objective (obj). The pump is sent collimated into the objective while the probe is focused in the back focal plane of the objective to result in confocal and widefield excitation spots, respectively. Light reflected from the sample-substrate interface or scattered from the sample is collected, spectrally filtered (through a longpass and bandpass filter, BP) and focused onto a detector (CMOS). An aperture (Ap) may be used block the scattered light and image primarily normally-reflected light during alignment/testing stages.

Computational methods: We calculate the electronic structure of $\mathrm{Ag}-\mathrm{Bi}$ and $\mathrm{Ag}-\mathrm{Tl}$ with density functional theory (DFT), as implemented in the Quantum Espresso computational package ${ }^{6}$. For our DFT calculations, we use the PBE0 global hybrid functional ${ }^{7}$. We use norm conserving PBE pseudopotentials provided in the Pseudo Dojo database ${ }^{8}$, a plane wave cutoff of $100 \mathrm{Ry}$, and a $4 \times 4 \times 4 \mathbf{k}$ and q-point grid for both the charge density and the exact exchange, respectively. In the case of $\mathrm{Ag}-\mathrm{Bi}$, the k-point mesh used in our calculations is centered at the $\Gamma$ point, while for $\mathrm{Ag}-\mathrm{Tl}$ we use a half-shifted grid. This latter choice was made in order to efficiently correct the unphysical conduction and valence band crossing calculated with non-hybrid DFT-PBE for Ag-TI².

In order to compute the electronic band structure and effective masses, we interpolate the DFT-PBEO generalized Kohn-Sham eigenvalues using Wannier functions with the Wannier90 code ${ }^{9}$. We calculate maximally localized Wannier functions ${ }^{10,11}$ using the $\mathrm{Bi}-\mathrm{p}, \mathrm{Br}-\mathrm{p}, \mathrm{Ag}-\mathrm{d}$, and $\mathrm{Tl}-\mathrm{s}$ as starting projections for $\mathrm{Ag}-\mathrm{Bi}$ and $\mathrm{Ag}-\mathrm{Tl}$, respectively. We generate the effective mass tensor as the inverse of the Hessian matrix obtained from second order partial derivatives of the energy eigenvalues at the conduction band minimum, with respect to the wave vector in all Cartesian directions. We perform the second partial derivatives around the $\mathrm{L}$ and $\Gamma$-points for $\mathrm{Ag}-\mathrm{Bi}$ and $\mathrm{Ag}-\mathrm{Tl}$, respectively, using the finite difference method, with a wave-vector increment of $0.02 \AA^{-1}$. 


\section{Ag-Bi carrier dynamics}
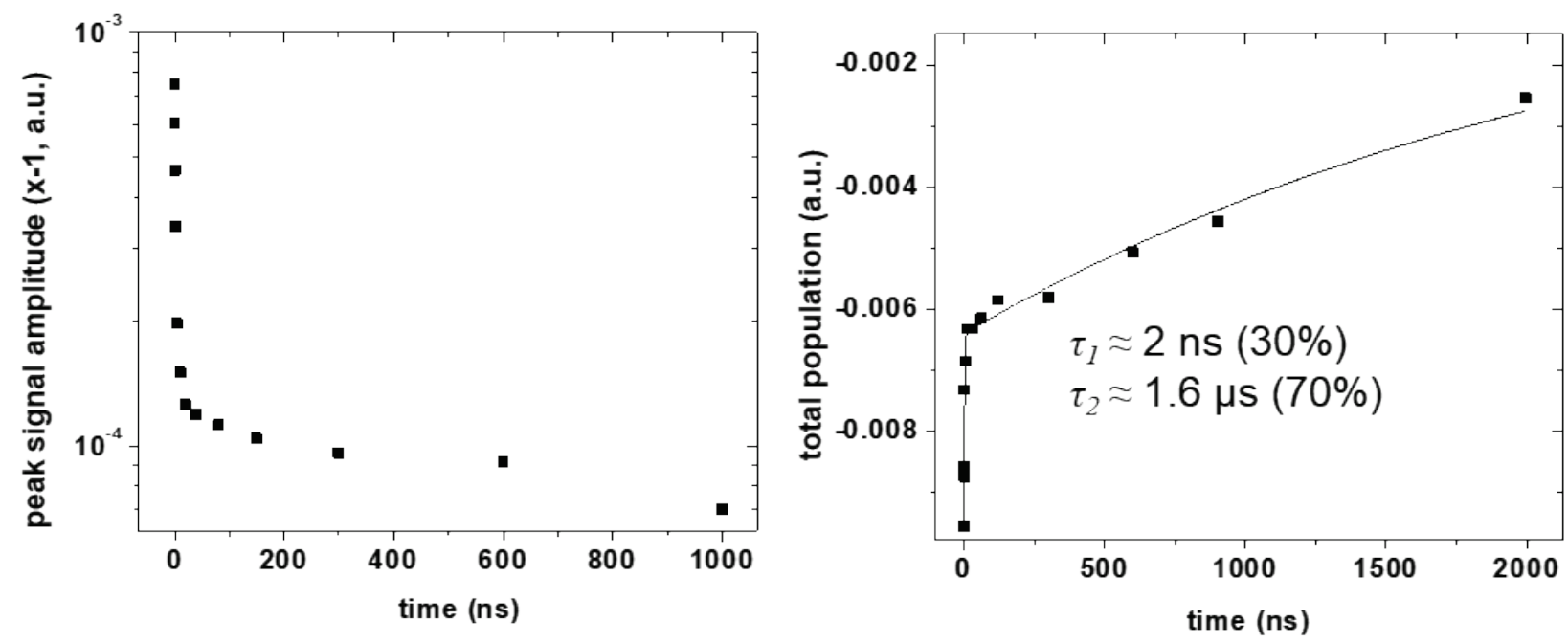

Figure S2. Peak signal amplitude (left) and total population, i.e. spatially integrated signal, (right) kinetics from stroboSCAT measurements ( $440 \mathrm{~nm}$ pump, $640 \mathrm{~nm}$ probe) of as-made Ag-Bi single crystals. The peak signal amplitude is extracted from the amplitude of Gaussian fits to the raw stroboSCAT images. These kinetics report on the carrier density decay as a function of carrier recombination and diffusion out of the initially focused excitation spot. The total population decay is estimated by integrating over the signal. The latter displays two population regimes, one on few-ns timescale and one on microsecond timescales, as has previously been observed in $\mathrm{Ag}-\mathrm{Bi}^{1,12}$. 


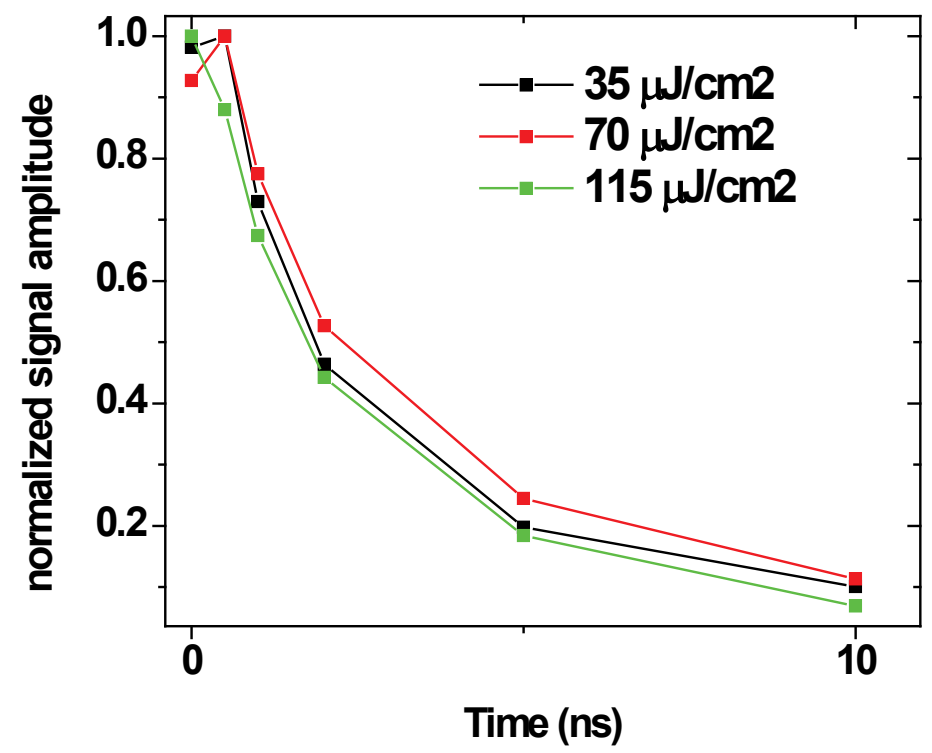

Figure S3. Normalized signal amplitudes extracted from stroboSCAT measurements (440 nm pump, 640 $\mathrm{nm}$ probe) of as-made Ag-Bi single crystals at different pump fluences. To ensure that lateral and axial diffusion contribute minimally to the observed signal, we restrict this analysis to short pump-probe time delays, over which diffusion out of the finite excitation spot is negligible. The excitation spot is made larger ( 700 nm FWHM) by irising out the pump prior to entering the objective to further reduce diffusion contributions. Large peak carrier densities at early times ensure the highest possible sensitivity to nonlinear dynamics. If nonlinear recombination pathways were present due to carrier-carrier interactions (e.g. Auger recombination) at these fluences, the early-time dynamics would strongly depend on pump fluence. The obtained carrier dynamics appear to be fluence-independent over the tested range, as was previously observed in transient absorption measurements ${ }^{12}$. The fluence used for the data shown in the main text is $66 \mu \mathrm{J} / \mathrm{cm}^{2}$, well within a linear excitation regime. 


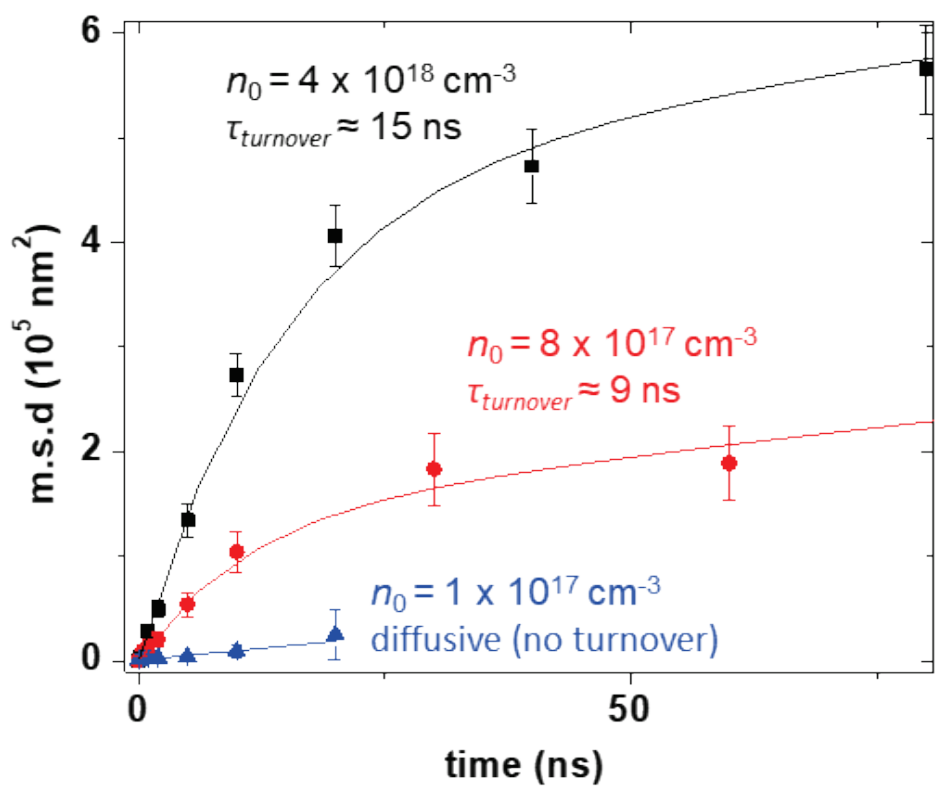

Figure S4. m.s.d. extracted from stroboSCAT measurements ( $440 \mathrm{~nm}$ pump, $640 \mathrm{~nm}$ probe) of as-made $\mathrm{Ag}$-Bi single crystals at different initial carrier densities $\left(n_{0}\right)$. As the initial excitation density decreases, the turnover from fast to slow diffusivity occurs earlier. When the initial density drops to below the near-surface trap density (estimated to be $\sim 2 \times 10^{17} \mathrm{~cm}^{-3}$, see main text), the diffusivity becomes linear. The signal to noise ratio at low excitation fluences is too small to enable thorough analyses of diffusivity behavior. Nevertheless, the observed trend qualitatively supports our hypothesis that the turnover from fast to slow diffusivity stems from a trap-saturated regime at early times. $D_{\text {trapped }}$ in the three measured excitation regimes are fairly similar, as expected: $D_{\text {trapped }}=0.007 \pm 0.001,0.005 \pm 0.001$, and $0.008 \pm$ $0.002 \mathrm{~cm}^{2} / \mathrm{s}$ for the high, medium and low excitation densities measured, respectively. 


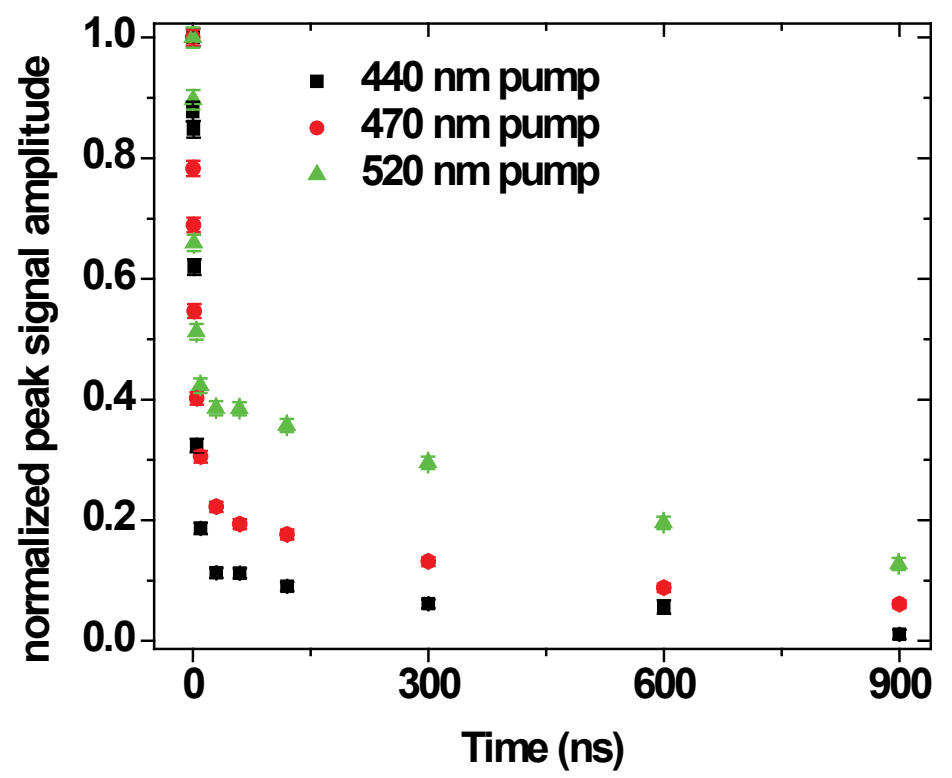

Figure S5. Pump-excitation-wavelength dependence of carrier dynamics in as-made Ag-Bi single crystals. The fast early-time dynamics are thought to arise primarily due to surface-mediated recombination ${ }^{1,13}$. Ag-Bi's absorptivity drops by a factor of 8 across the 440-520 nm range. As a result, lower-energy excitation penetrates deeper into the material, generating a larger ratio of bulk/surface carriers. Based on measured absorption coefficients ${ }^{13}$, we estimate penetration depths of $35 \mathrm{~nm}, 70 \mathrm{~nm}$ and $280 \mathrm{~nm}$ for 440, 470 and $520 \mathrm{~nm}$ pumps, respectively. Our stroboSCAT measurements concur with previous measurements showing a higher proportion of long-lived carriers when the bulk/surface carrier ratio is larger, thus suggesting that bulk carriers are longer-lived than surface carriers ${ }^{1,13}$. 


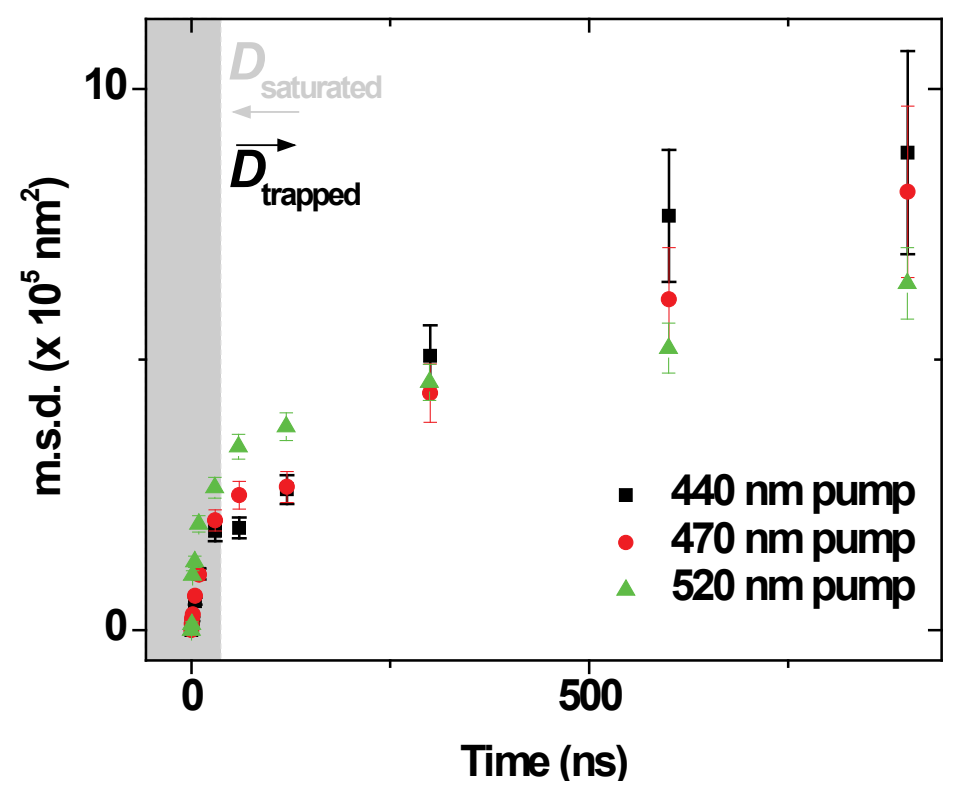

Figure S6. Pump-excitation-wavelength dependence of mean squared displacement measured with stroboSCAT in Ag-Bi. The measurements show a larger $D_{\text {saturated }}$ with lower-energy excitation (greater bulk/surface carrier ratio), and a turnover from $D_{\text {saturated }}$ to $D_{\text {trapped }}$ that occurs at lower carrier densities. These measurements confirm three aspects of our analysis: the fast initial diffusivity $D_{\text {saturated }}$ is not due to nonlinear carrier dynamics at the crystal surface, which would be minimized with lower-energy excitation (Figure S5) and thus would result in a lower contribution of $D_{\text {saturated }}$ to the $520 \mathrm{~nm}$ signal, opposite to what we observe; the trap density at the surface is lower in the bulk than at the surface, as evidenced by a turnover from $D_{\text {saturated }}$ to $D_{\text {trapped }}$ occurring at lower carrier densities with lower-energy excitation; and lower trap densities lead to larger $D_{\text {saturated }}$ despite occurring in the trap-saturated regime, i.e. filled traps contribute to lattice disorder which reduces $D_{\text {saturated. }}$.

\section{Ag-Tl carrier dynamics}
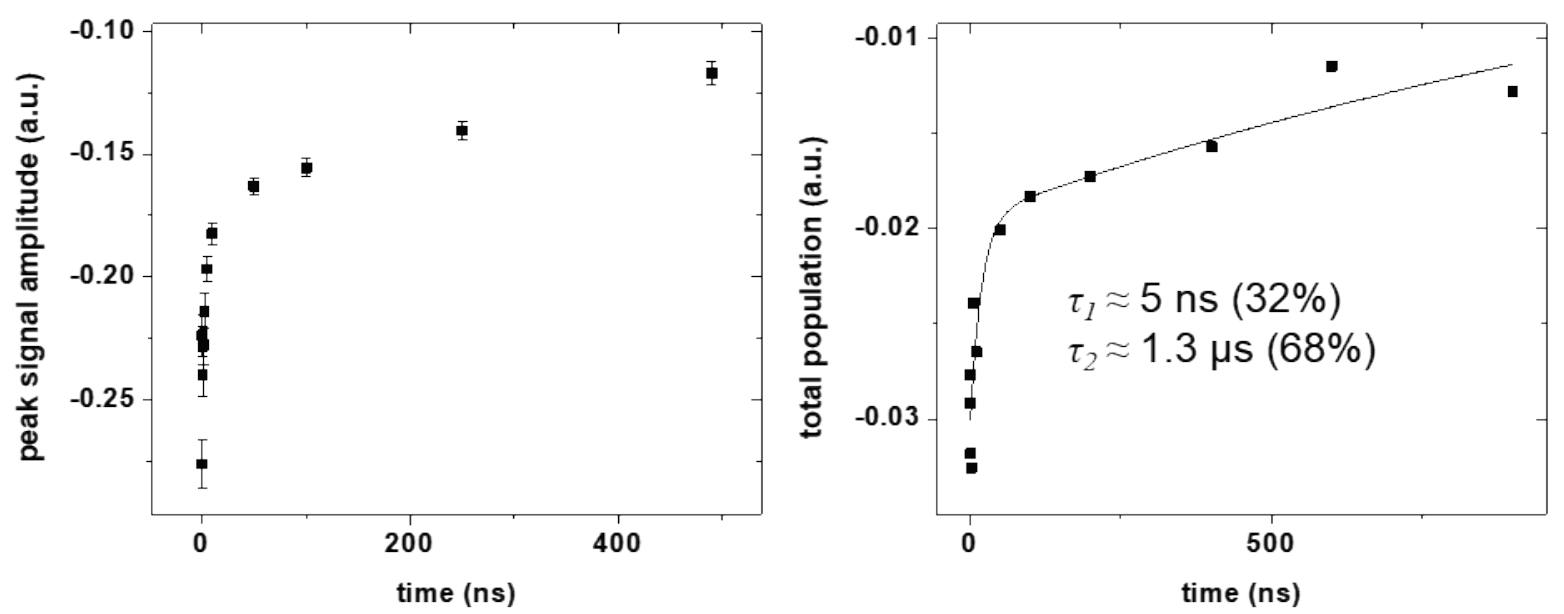

Figure S7. Peak signal amplitude (left) and total (integrated) population (right) kinetics from stroboSCAT measurements (440 $\mathrm{nm}$ pump, $640 \mathrm{~nm}$ probe) of as-made Ag-Tl single crystals. 


\section{Supplementary references}

(1) Slavney, A. H.; Hu, T.; Lindenberg, A. M.; Karunadasa, H. I. A Bismuth-Halide Double Perovskite with Long Carrier Recombination Lifetime for Photovoltaic Applications. J. Am. Chem. Soc. 2016, 138, 2138-2141.

(2) Slavney, A. H.; Leppert, L.; Saldivar Valdes, A.; Bartesaghi, D.; Savenije, T. J.; Neaton, J. B.; Karunadasa, H. I. Small-Band-Gap Halide Double Perovskites. Angew. Chemie Int. Ed. 2018, 57, 12765-12770.

(3) Pan, W.; Wu, H.; Luo, J.; Deng, Z.; Ge, C.; Chen, C.; Jiang, X.; Yin, W.-J.; Niu, G.; Zhu, L.; Yin, L.; Zhou, Y.; Xie, Q.; Ke, X.; Sui, M.; Tang, J. Cs2AgBiBr6 Single-Crystal X-Ray Detectors with a Low Detection Limit. Nat. Photonics 2017, 11, 726-732.

(4) Delor, M.; Weaver, H. L.; Yu, Q.; Ginsberg, N. S. Imaging Material Functionality through ThreeDimensional Nanoscale Tracking of Energy Flow. Nat. Mater. 2020, 19, 56-62.

(5) Ortega Arroyo, J.; Cole, D.; Kukura, P. Interferometric Scattering Microscopy and Its Combination with Single-Molecule Fluorescence Imaging. Nat. Protoc. 2016, 11, 617-633.

(6) Giannozzi, P.; Andreussi, O.; Brumme, T.; Bunau, O.; Buongiorno Nardelli, M.; Calandra, M.; Car, R.; Cavazzoni, C.; Ceresoli, D.; Cococcioni, M.; Colonna, N.; Carnimeo, I.; Dal Corso, A.; de Gironcoli, S.; Delugas, P.; DiStasio, R. A.; Ferretti, A.; Floris, A.; Fratesi, G.; Fugallo, G.; Gebauer, R.; Gerstmann, U.; Giustino, F.; Gorni, T.; Jia, J.; Kawamura, M.; Ko, H.-Y.; Kokalj, A.; Küçükbenli, E.; Lazzeri, M.; Marsili, M.; Marzari, N.; Mauri, F.; Nguyen, N. L.; Nguyen, H.-V.; Otero-de-la-Roza, A.; Paulatto, L.; Poncé, S.; Rocca, D.; Sabatini, R.; Santra, B.; Schlipf, M.; Seitsonen, A. P.; Smogunov, A.; Timrov, I.; Thonhauser, T.; Umari, P.; Vast, N.; Wu, X.; Baroni, S. Advanced Capabilities for Materials Modelling with Quantum ESPRESSO. J. Phys. Condens. Matter 2017, 29, 465901.

(7) Perdew, J. P.; Ernzerhof, M.; Burke, K. Rationale for Mixing Exact Exchange with Density Functional Approximations. J. Chem. Phys. 1996, 105, 9982-9985.

(8) van Setten, M. J.; Giantomassi, M.; Bousquet, E.; Verstraete, M. J.; Hamann, D. R.; Gonze, X.; Rignanese, G. M. The PSEUDODOJO: Training and Grading a 85 Element Optimized NormConserving Pseudopotential Table. Comput. Phys. Commun. 2018, 226, 39-54.

(9) Mostofi, A. A.; Yates, J. R.; Pizzi, G.; Lee, Y. S.; Souza, I.; Vanderbilt, D.; Marzari, N. An Updated Version of Wannier90: A Tool for Obtaining Maximally-Localised Wannier Functions. Comput. Phys. Commun. 2014, 185, 2309-2310.

(10) Marzari, N.; Vanderbilt, D. Maximally Localized Generalized Wannier Functions for Composite Energy Bands. Phys. Rev. B 1997, 56, 12847-12865.

(11) Souza, I.; Marzari, N.; Vanderbilt, D. Maximally Localized Wannier Functions for Entangled Energy Bands. Phys. Rev. B - Condens. Matter Mater. Phys. 2002, 65, 1-13.

(12) Hoye, R. L. Z.; Eyre, L.; Wei, F.; Brivio, F.; Sadhanala, A.; Sun, S.; Li, W.; Zhang, K. H. L.; MacManusDriscoll, J. L.; Bristowe, P. D.; Friend, R. H.; Cheetham, A. K.; Deschler, F. Fundamental Carrier Lifetime Exceeding $1 \mathrm{Ms}$ in Cs2AgBiBr6 Double Perovskite. Adv. Mater. Interfaces 2018, 5, 2-9.

(13) Bartesaghi, D.; Slavney, A. H.; Gélvez-Rueda, M. C.; Connor, B. A.; Grozema, F. C.; Karunadasa, H. I.; Savenije, T. J. Charge Carrier Dynamics in Cs2AgBiBr6 Double Perovskite. J. Phys. Chem. C 2018, 122, 4809-4816. 